\title{
SOBRECARGA DOS CUIDADORES DE IDOSOS: RELATO DE EXPERIÊNCIA
}

OVERLOAD OF CAREGIVERS OF THE ELDERLY: EXPERIENCE REPORT

SOBRECARGA DE LOS CUIDADORES DE ANCIANOS: RELATO DE EXPERIENCIA

Gabriela Medeiros Steindorff ${ }^{1}$

Sidnei Batista de Oliveira Junior 2

Diogo da Rosa Viana ${ }^{3}$

João Nunes Maidana Júnior 4

Cenir Gonçalves Tier ${ }^{5}$

Vanessa Alvez Mora da Silva ${ }^{6}$

Palavras-chave:

Cuidadores; Enfermagem em Saúde Comunitária; Serviços de Saúde para Idosos; Visita Domiciliar.

Keywords: Caregivers; Community Health Nursing; Health Services for the Elderly; House calls.

Palabras clave: Cuidadores; Enfermería en Salud Comunitaria; Servicios de Salud para Ancianos; Visita Domiciliaria.

Submetido: $15 / 09 / 2017$

Aprovado: $16 / 03 / 2018$

Autor(a) para Correspondência: Gabriela Medeiros Steindorff End: Rua Santana $n^{\circ} 4547$, Bairro Santana, CEP: 97502-214 Uruguaiana Rio Grande de Sul - Brasil E-mail: gsteindorff20@gmail.com

\section{RESUMO}

Este artigo descreve a experiência de estudantes de Enfermagem no que se refere à sobrecarga do cuidador de idosos. Trata-se de relato de experiencia cujo cenário consistiu no domicílio de idosos de um município de médio porte na região da Fronteira Oeste do Rio Grande do Sul, com visitas domiciliares aos idosos. As visitas foram realizadas em 3 encontros, em abril e maio de 2017, com 7 famílias de idosos indicadas por Agentes Comunitários de Saúde (ACS) da Estratégia Saúde da Família (ESF). 0 cuidador do idoso se mostra fundamental para sanar dúvidas da equipe de saúde. Nas 7 famílias visitadas, os cuidadores, todos do sexo feminino, relataram sentir-se sozinhos no cuidado ao idoso. Observou-se necessidade de: monitorar o uso de medicação e a pressão arterial; realizar consultas periódicas; e proporcionar ajuda nas atividades diárias. Nas visitas domiciliares, constatou-se que a sobrecarga do cuidador assume diferentes formas, tanto psicológicas como físicas. Nesse sentido, mostra-se necessário que os profissionais da saúde tenham um olhar voltado à integralidade do cuidado ao idoso, que também engloba a atenção à saúde do cuidador.

1. Estudante de Graduação em Enfermagem na Universidade Federal do Pampa (Unipampa). Uruguaiana (RS), Brasil. E-mail: gsteindorff20@gmail.com

2. Estudante de Graduação em Enfermagem na Unipampa. Uruguaiana (RS), Brasil. E-mail: juniorboj30@gmail. com

3. Enfermeiro graduado pela Unipampa.Uruguaiana (RS),Brasil. E-mail: diogoviana95@yahoo.com.br

4. Enfermeiro graduado pela Unipampa. Uruguaiana (RS), Brasil. E-mail: juniordana@hotmail.com

5. Enfermeira. Doutora em Enfermagem. Professora na Unipampa. Uruguaiana (RS), Brasil. E-mail: cgtier@ hotmail.com

6. Enfermeira. Mestre em Ciências da Saúde. Professora na Unipampa. Uruguaiana (RS), Brasil. E-mail: valvez_ enfermagem@hotmail.com 


\section{ABSTRACT}

This article describes the experience of Nursing students regarding the overload of caregivers of the elderly. This is an experience report whose scenario consisted of the elderly's household in a medium-sized municipality in the Fronteira Oeste region of Rio Grande do Sul, Brazil, with house calls to the elderly. The visits were carried out in 3 meetings, in April and May 2017, with 7 families of the elderly indicated by Community Health Workers (CHWs) from the Family Health Strategy (FHS). The caregiver of the elderly is crucial to solve the health team's doubts. In the 7 families visited, the caregivers, all of them women, reported feeling alone when caring for the elderly. There was a need to: monitor medication use and blood pressure; conduct regular appointments; and provide help in daily living activities. In the house calls, it was found that the caregiver's overload takes various forms, both psychological and physical. Thus, it is necessary that health professionals have a focus on comprehensive care for the elderly, which also encompasses providing the caregiver with care.

\section{RESUMEN}

Este artículo describe la experiencia de estudiantes de Enfermería con respecto a la sobrecarga del cuidador de ancianos. Este es un informe de experiencia cuyo escenario consistió en el hogar de ancianos en un municipio de tamaño medio en la región de la Fronteira Oeste de Rio Grande do Sul, Brasil, con visitas domiciliarias a los ancianos. Las visitas se llevaron a cabo en 3 reuniones, en abril y mayo de 2017, con 7 familias de ancianos indicadas por Agentes Comunitarios de Salud (ACS) de la Estrategia Salud de la Familia (ESF). El cuidador del anciano es crucial para resolver dudas del equipo de salud. En las 7 familias visitadas, los cuidadores, todos del sexo femenino, informaron sentirse solos cuando cuidaban al anciano. Hubo una necesidad de: monitorear el uso de medicación y la presión arterial; realizar consultas regulares; y proporcionar ayuda en las actividades de la vida diaria. En las visitas domiciliarias, se constató que la sobrecarga del cuidador toma diversas formas, tanto psicológicas como físicas. En este sentido, es necesario que los profesionales de salud se centren en la atención integral para los ancianos, lo que también incluye brindar atención al cuidador.

\section{INTRODUÇÃO}

0 Brasil é um país que envelhece a passos largos. As alterações na pirâmide populacional são claras, inequívocas e irreversíveis. Nos últimos anos, é entre a população idosa que temos observado as taxas mais altas de crescimento populacional ${ }^{1}$.

0 Instituto Brasileiro de Geografia e Estatística (IBGE) corrobora essa afirmativa. Em 2016, os indivíduos acima de 65 anos representavam $8,1 \%$ da população no Brasil e a estimativa para 2030 é de que representarão $13,4 \%^{2}$.

Atualmente, a Atenção Primária à Saúde (APS) desempenha importante papel em relação à população idosa, pois se caracteriza por um conjunto de ações de saúde, no âmbito individual e coletivo, que abrange a promoção e a manutenção da saúde para proporcionar uma atenção integral que aumente a autonomia das pessoas e melhore seus determinantes e condicionantes de saúde ${ }^{3}$.

Sabe-se que, para dar conta de toda essa amplitude da APS, surgiu em 1994 o Programa de Saúde da Família (PSF) - conhecido atualmente como Estratégia Saúde da Família (ESF) -, que busca reorientar o modelo assistencial, antes biomédico, com o intuito de formar equipes de saúde multi e transdisciplinares ${ }^{4}$. Tais mudanças exigem cada vez mais que as equipes da ESF busquem estratégias diferentes na abordagem a toda a população atendida, mais especificamente aos idosos, tendo em vista que a APS é a porta de entrada no Sistema Único de Saúde (SUS) e que ela se fortalece cada vez mais em âmbito nacional, captando e acompanhando os idosos para garantir a equidade e integralidade das ações de saúde voltadas a eles ${ }^{5}$.

Nessa perspectiva, a Política Nacional do Idoso surgiu como uma estratégia/ferramenta de fomento dos direitos sociais dessa população, buscando um cuidado integral tanto do indivíduo como de sua família ${ }^{6}$. É de suma importância que se conheça o contexto social desse idoso e seu relacionamento com os cuidadores para que a equipe da APS possa traçar estratégias segundo as necessidades de saúde de cada indivíduo. Assim, a caderneta de saúde da pessoa idosa é um instrumento auxiliar para uma adequada atenção à saúde do idoso, usado tanto pelas equipes de saúde com pelos idosos e seus familiares e cuidadores ${ }^{7}$.

Logo, quanto maior o grau de fragilidade, maior 0 nível de dependência funcional do idoso, que demandará cuidados - formais ou informais. 
Desse modo, a sobrecarga do cuidador do idoso é algo comum, principalmente devido ao seu despreparo para desempenhar essa função, e isso pode causar problemas de saúde, tanto psicológicos como físicos ${ }^{8}$. Nesse contexto, mostra-se fundamental o apoio dos profissionais da saúde ao cuidador do idoso.

A sobrecarga pode implicar graves consequências tanto para o cuidador (formal ou informal) como para o idoso e sua família. A falta de suporte e orientação adequada para as ações de cuidado aumenta a possibilidade de adoecimento do cuidador.

Considera-se cuidador informal alguém que reside no mesmo local do idoso, geralmente um membro da família. Já o cuidador formal recebe um salário e geralmente se desloca para sua casa ao final de seu expediente. A atenção de enfermagem voltada à saúde da família e à saúde do idoso deve pautar-se pela compreensão das crenças e dos significados apresentados pela realidade social e familiar. Buscase romper tabus e preconceitos para auxiliar na formulação de estratégias para proporcionar cuidado integral. 0 foco do cuidado ao idoso acaba sendo individual, mas advém de construção e interação com a família buscando o bem-estar comum ${ }^{9}$.

A enfermagem deve repensar suas atitudes diante do modelo biomédico de cuidado, revendo conceitos e abordagens ao idoso e ao cuidador. A pessoa deve ser entendida como um ser individual e coletivo, participante ativo de uma comunidade - que deve respeitar seus significados e sua liberdade de ação e tomada de decisão em sua vida e nos cuidados recebidos ${ }^{9}$.

Por sua vez, alguns profissionais da saúde ainda apresentam uma visão de cuidado limitado à assistência técnica, fato que revela a necessidade de expandir o conhecimento dos aspectos conceituais do cuidado para além das obrigações funcionais ${ }^{6}$ - que incluem as orientações para garantir uma atenção também focada na saúde mental do cuidador.

Diante do exposto, este artigo descreve a experiência de estudantes de graduação em Enfermagem no que se refere à sobrecarga do cuidador de idosos, com a realização de visitas domiciliares.

\section{METODOLOGIA}

Trata-se de um relato de experiência que descreve a vivência de estudantes durante as atividades práticas da disciplina "Cuidado à Saúde do Idoso", no âmbito do curso de Graduação em Enfermagem da Universidade Federal do Pampa (Unipampa).

\section{...alguns profissionais da saúde \\ ainda apresentam uma \\ visão de cuidado \\ limitado à assistência \\ técnica...}

0 cenário consistiu no domicílio de idosos de um município de médio porte na região da Fronteira Oeste do Rio Grande do Sul, com visitas domiciliares de uma equipe da ESF - para preencher a caderneta de saúde do idoso n. 19. Essa vivência ocorreu em 3 encontros, em abril e maio de 2017, realizados com 7 famílias adscritas no território de uma unidade da ESF localizada na zona urbana do município em questão.

Essa unidade da ESF possui 2 equipes, compostas por 2 enfermeiros, 2 médicos generalistas, 3 técnicos de enfermagem, 1 nutricionista, 1 educador físico e 8 Agentes Comunitários de Saúde (ACS). A área de abrangência desse serviço de saúde é composta por 13 microáreas, atendendo no território, de acordo com o Sistema de Informações da Atenção Básica (Siab), uma população de 8.800 pessoas, das quais cerca de $35 \%$ têm 60 anos ou mais.

Nesse serviço, o atendimento à comunidade ocorre nos turnos matutino e vespertino, respectivamente, das $7: 30$ às $11: 30$ e das $13: 30$ às $17: 30$, estruturandose a partir do acolhimento à livre demanda, segundo critérios da classificação de risco.

Ressalta-se que os domicílios que constituíram o cenário deste relato foram indicados por um ACS - um dos principais personagens na implementação do SUS, pois fortalece a integração entre os serviços de saúde da ESF e a comunidade.

\section{RESULTADOS E DISCUSSÃO}

Os enfermeiros que atuam no âmbito da APS devem identificar casos de sobrecarga do cuidador de idosos e propor ações de cuidado ao cuidador que contribuam para a manutenção de sua saúde física e mental e de sua qualidade de vida ${ }^{10}$.

Durante as práticas da disciplina "Cuidado à Saúde do Idoso", a proposta das visitas domiciliares surgiu com a finalidade de conhecer a população idosa na área de abrangência da unidade da ESF, para preencher sua caderneta do idoso. As residências foram 
indicadas e selecionadas conforme o cadastro dos ACS. As visitas domiciliares por parte dos estudantes de graduação em Enfermagem se mostram importantes por proporcionar o diálogo e o levantamento da população idosa em foco.

Uma das ferramentas mais expressivas das unidades da ESF são as visitas domiciliares, pois constituem momentos de integração entre os serviços de saúde e a população, fixando e fortalecendo o vínculo entre a equipe multidisciplinar de saúde e os usuários da APS ${ }^{11}$.

Essa aproximação também visa a acompanhar as necessidades de saúde da comunidade, zelando pelas condições de vida adequadas (saneamento, higiene, moradia etc.) no território ${ }^{12}$.

Treinamento ou experiência insuficiente e falta de conhecimento para a visita domiciliar podem influenciar o cuidado domiciliar, gerando desafios para a atuação do enfermeiro, que podem ser amenizados por meio de iniciativas de formação profissional13. Assim, necessita-se cada vez mais trabalhar sob a perspectiva de capacitar esses profissionais para a visita domiciliar.

Durante as visitas domiciliares, identificou-se que o papel do cuidador é fundamental para sanar dúvidas da equipe de saúde sobre o idoso, pois ele se apresenta como um sujeito conhecedor da vida e das necessidades desse idoso. Durante o preenchimento da caderneta do idoso, em vários momentos os pesquisadores necessitaram da ajuda do cuidador para responder algumas questões, principalmente no que se refere às medicações e à alimentação. Entretanto, todos os cuidadores relatam sobrecarga emocional e física, mencionando que, no cuidado ao idoso, as dúvidas e incertezas ficam sob sua responsabilidade e que geralmente vivenciam esses momentos sozinhos.

A literatura científica alerta que o cuidador, com o sofrimento decorrente de tais dúvidas e incertezas, necessita do apoio da equipe multidisciplinar de saúde para auxiliá-lo a conduzir as atividades corretas referentes ao idoso ${ }^{14}$.

Todos os cuidadores que participaram do estudo relataram sentir-se responsáveis tanto pela saúde do idoso como pelo manejo de suas limitações físicas e emocionais. Durante os diálogos, eles declararam que sempre buscam conversar com o idoso sobre a importância de manter seu cérebro ativo e de adotar bons hábitos de alimentação.

Um estudo científico valida tais achados e conclui ser fundamental para os profissionais da saúde o

\section{...a saúde do cuidador, tanto física como mental, afeta o cuidado e a saúde do idoso.}

acompanhamento de um cuidador de idosos para atender plenamente as necessidades dos idosos ${ }^{14}$.

Todos os cuidadores que participaram deste relato de experiência tinham vínculo familiar com os idosos e eram do sexo feminino - esses indivíduos relataram sentir-se sozinhos no cuidado a idoso, carecendo de ajuda dos demais membros da família.

Integrar a família e o idoso de modo harmonioso constitui um desafio. Para que isso aconteça, é preciso intervir no sofrimento familiar com foco em suas singularidades. As práticas profissionais no setor saúde demandam cada vez mais capacitação para compreender e atender as necessidades de saúde individuais e promover a cooperação entre os membros da família ${ }^{15}$.

0 membro da família que assume a função de cuidador deve adquirir novos hábitos. Sua vida se transforma por completo - desde assuntos menos relevantes do cotidiano até temas significativos, como privacidade, férias e viagens. Tais mudanças potencializam cada vez mais a sobrecarga do cuidador ${ }^{14}$.

Neste estudo, os cuidadores relataram sentir-se angustiados e tristes com a própria vida diariamente e identificam-se com sintomas de depressão. A grande maioria já buscou ajuda voltada à sua saúde mental e faz uso de medicação, porém, não apontam melhora de seu quadro nem significância no acompanhamento desses agravos psicológicos.

É essencial incluir a avaliação do sofrimento psíquico do cuidador, a fim de detectar distúrbios mentais precocemente. Sabe-se que a saúde do cuidador, tanto física como mental, afeta o cuidado e a saúde do idoso ${ }^{16}$.

Nos diálogos, além de sobrecarga emocional, os cuidadores indicaram limitações físicas decorrentes de dores osteomusculares, causadas tanto pelos afazeres domésticos como pela dependência física do idoso. Ademais, esses cuidadores não têm vínculo com a ESF, não frequentam grupos e sentem-se desestimulados a cuidar de sua própria saúde.

A participação em grupos de saúde melhora 
questões relacionadas tanto à saúde física como à saúde mental, tendo em vista que assumem o papel de estimuladores de uma vida mais saudável e promovem a autoestima, proporcionando a inclusão no meio social e evitando o isolamento ${ }^{11}$.

Enfatiza-se a importância dos grupos de atividades físicas de idosos, bem como a participação de seus cuidadores, pois melhoram a aptidão funcional, que é vital para a vida cotidiana. Almeja-se que os idosos sejam capazes de realizar suas atividades diárias com esforço muscular, condicionamento físico e equilíbrio ${ }^{17}$. Esses grupos de atividades físicas constituem uma ferramenta de vínculo entre o idoso e seu cuidador e a ESF.

É necessário que o enfermeiro tenha sensibilidade no momento do diálogo com esses cuidadores; quando necessário, deve-se ter conhecimento acerca do modo de identificar a sobrecarga desses indivíduos, por exemplo, por meio de uma escala ou outro recurso de avaliação.

0 inventário de carga do cuidador é um exemplo de escala que pode ser aplicada na ESF. Esse instrumento avalia a carga do cuidador e fornece informações valiosas sobre o impacto dos idosos em vários domínios da vida dos cuidadores e familiares, podendo ajudar os enfermeiros a desenvolver intervenções para prevenir problemas de saúde entre os cuidadores e melhorar sua qualidade de vida ${ }^{18}$.

Ressalta-se que também existem escalas para cuidadores informais aplicáveis na APS, por exemplo, - Questionário de Avaliação da Sobrecarga do Cuidador Informal, que avalia a qualidade de vida relacionada aos aspectos físicos, emocionais e sociais da saúde ${ }^{10}$.

Observam-se dificuldades para que a equipe de saúde entenda a proposta de integralidade do cuidado. Neste estudo, identificou-se que os ACS limitam seu foco ao idoso, não englobando a saúde de sua família - a maioria das questões para 0 cuidador se referem à saúde do idoso.

Outro estudo semelhante comprova essa afirmação, pois conclui que alguns profissionais valorizam e fazem uso da tecnologia relacional, alicerçada em acolhimento, vínculo, interação etc. Em algumas unidades da ESF, contudo, ainda predomina a visão biologicista do cuidado, com ênfase em procedimentos, normas e medicamentos ${ }^{19}$.

0 embasamento científico alerta os enfermeiros quanto às questões do acolhimento em suas ações, evidenciando que a reorganização de seu processo de trabalho depende somente de fatores externos e

\section{...grupos de atividades físicas constituem uma ferramenta de vínculo entre o idoso e seu cuidador e a ESF.}

desconsidera seu compromisso com o cuidado humanizado ${ }^{20}$. Portanto, deve-se discutir cada vez mais a necessidade de manter os vínculos profissionalusuários, o acolhimento e a escuta.

Assim, a visita domiciliar se torna um espaço de escuta e de diálogo e um momento de acolhimento e de criação de vínculo, proporcionando novos modos de cuidar - mais humanos e mais acolhedores, ao envolver a afetividade e os laços de confiança entre profissionais, usuários, família e comunidade ${ }^{21}$.

Durante a escuta, os pesquisadores observaram que quanto maior o grau de dependência desses idosos, mais frequentes os relatos de sobrecarga dos cuidadores. Dentre os tipos de dependência, constatase a necessidade de monitorar o uso de medicação e a pressão arterial; realizar consultas periódicas; e proporcionar ajuda nas atividades diárias, como vestir roupas e tomar banho.

Nesse sentido, há maior sobrecarga dos cuidadores de idosos que necessitam de apoio para lidar com as morbidades, o que aumenta o nível de dependência do idoso e, por conseguinte, a demanda de cuidado. Por isso, quanto maior a dependência do idoso, maior a sobrecarga do cuidador ${ }^{22}$.

As visitas domiciliares disponibilizam momentos para que os pesquisadores ofereçam educação em saúde, por meio de orientações sobre alimentação, quedas, doenças crônicas e saúde mental, assim como de fortalecimento do vínculo com a ESF e entre o profissional e o usuário.

0 risco de queda entre idosos serve de alerta para os profissionais observarem fatores como a prevalência do sexo feminino, a má qualidade de sono e a baixa força muscular23. Torna-se imprescindivel que os enfermeiros estejam cientes de tais fatores para fins de prevenção, podendo ser abordados nas visitas domiciliares.

Considerando as características do processo de envelhecimento, com suas múltiplas facetas, as fragilidades envolvidas e os níveis de independência dos idosos, há necessidade de intervenções de outros 
campos além do setor saúde para o desenvolvimento de estratégias que melhorem o cuidado ao idoso ${ }^{24}$, portanto, deve-se trabalhar cada vez mais sob a perspectiva multiprofissional na APS.

É essencial que o enfermeiro dedique atenção ao contexto do cuidado ao idoso como um todo ao planejar ações de saúde voltadas a essa população.

\section{CONCLUSÃO}

Vivenciar as complexidades da saúde do idoso na APS se mostra de vital importância para a formação acadêmica em Enfermagem, com vistas à reflexão acerca da atuação dos profissionais da saúde diante da sobrecarga do cuidador de idosos.

Nas visitas domiciliares, identificou-se que tal sobrecarga assume diferentes formas, tanto psicológicas como físicas. Além de sintomas relacionados à depressão, levantaram-se relatos de dores osteomusculares. Ademais, as visitas domiciliares caracterizam-se como um momento oportuno para a educação em saúde voltada à família como um todo.

Os obstáculos deste estudo consistiram no estabelecimento de vínculo com cada idoso e seus cuidadores em um único encontro, porém, tais dificuldades foram contornadas por meio do acolhimento e da escuta desses indivíduos. Há necessidade de maior discussão e reflexão acerca da visita domiciliar para que ela possa ser aperfeiçoada.

Sugerem-se novas pesquisas voltadas a essa temática, bem como novos estudos que acompanhem os tipos de sobrecarga que acometem os cuidadores. Considera-se necessário que os profissionais adotem um olhar voltado à integralidade do cuidado ao idoso - e isso inclui a atenção ao cuidador.

\section{CONTRIBUIÇÃO DOS AUTORES}

Gabriela Medeiros Steindorff contribuiu com a concepção e realização da pesquisa e a redação do manuscrito. Sidnei Batista de Oliveira Junior contribuiu com a realização da pesquisa e a redação do manuscrito. Diogo da Rosa Viana contribuiu com a redação e revisão crítica do manuscrito. João Nunes Maidana Júnior contribuiu com a redação e revisão crítica do manuscrito. Cenir Gonçalves Tier contribui com a revisão crítica do manuscrito. Vanessa Alvez Mora da Silva contribuiu com a revisão crítica do manuscrito.

\section{REFERÊNCIAS}

1. Kuchemann BA. Envelhecimento populacional, cuidado e cidadania: velhos dilemas e novos desafios. Sociedade e Estado [serial on the internet]. 2012 [cited 2017 Jul 7];27(1):165-80. Available from: http://www.scielo.br/pdf/ se/v27n1/09.pdf

2. Brasil. Projeção da população do Brasil e das unidades da Federação [document on the internet]. Rio de Janeiro: IBGE; 2017 [cited 2018 Apr 22]. Available from: https://www. ibge.gov.br/apps/populacao/projecao/

3. Brasil. Política Nacional de Atenção Básica. Brasília (DF): Ministério da Saúde; 2012.

4. Soratto J, Pires DEP, Dornelles S, Lorenzetti J. Family Health Strategy: a technological innovation in health. Texto \& Contexto Enferm [serial on the internet]. 2015 [cited 2017 Aug 6];24(2):584-92. Available from: http://www. scielo.br/pdf/tce/v24n2/0104-0707-tce-24-02-00584.pdf

5. Santos M, Mattos IE. Condições de vida e saúde da população idosa do município de Guaramiranga-CE. Epidemiol Serv Saúde [serial on the internet]. 2011 [cited 2017 Jul 7];20(2):193-201. Available from: https://www.arca.fiocruz. br/bitstream/icict/5029/2/1082.pdf

6. Stackfleth R, Diniz MA, Fhon JRS, Vendruscolo TRP, Whebe SCCF, Marques $S$, et al. Sobrecarga de trabalho em cuidadores de idosos fragilizados que vivem no domicílio. Acta Paul Enferm [serial on the internet]. 2012 [cited 2017 Jul 7];25(5):768-74. Available from: http://dx.doi. org/10.1590/S0103-21002012000500019

7. Morais JC, Santos KF, Andrade CG, Costa ICP, Brito FMB, Fernandes MGM. Significado de cuidado: o olhar de profissionais e idosos institucionalizados. Rev Enferm UFPE On Line [serial on the internet]. 2015 [cited 2017 Jul 7];9(Suppl 7):8937-45. Available from: file:///D:/10683-22613-1-PB.pdf

8. Brasil. Caderneta de saúde da pessoa idosa [document on the internet]. Brasília (DF): Ministério da Saúde; 2014 [cited 2018 Apr 22]. Available from: http://bvsms.saude. gov.br/bvs/publicacoes/caderneta saude pessoa idosa 3ed.pdf

9. Perseguino MG, Horta ALM, Ribeiro CA. A família frente a realidade do idoso de morar sozinho. Rev Bras Enferm [serial on the internet]. 2017 [cited 2017 Jul 7];70(2):235-41. Available from: http://www.scielo.br/pdf/reben/v70n2/ pt 0034-7167-reben-70-02-0235.pdf

10. Monteiro EA, Mazin SC, Dantas RAS. Questionário de avaliação da sobrecarga do cuidador informal: validação para o Brasil. Rev Bras Enferm [serial on the internet]. 2015 [cited 2017 Aug 1];68(3):421-8. Available from: http:// www.scielo.br/pdf/reben/v68n 3/0034-7167reben-68-03-0421.pdf 
11. Gomes MFP, Fracolli LA, Machado BC. Atenção domiciliar do enfermeiro na Estratégia Saúde da Família. Mundo Saúde [serial on the internet]. 2015 [cited 2017 Jul 7];39(4):4705. Available from: https://www.saocamilo-sp.br/pdf/ mundo saude/155572/A08.pdf

12. Kebian LVA, Acioli S. A visita domiciliar de enfermeiros e agentes comunitários de saúde da Estratégia Saúde da Família. Rev Eletrônica Enferm [serial on the internet]. 2014 [cited 2017 Jul 7];16(1):161-9. Available from: file:///D:/20260-126142-1-PB.pdf

13. Andrade AM, Silva KL, Seixas CT, Braga PP. Atuação do enfermeiro na atenção domiciliar: uma revisão integrativa da literatura. Rev Bras Enferm [serial on the internet]. 2017 [cited 2017 Aug 6];70(1):210-9. Available from: http:// www.scielo.br/pdf/reben/v70n1/0034-7167reben-70-01-0210.pdf

14. Reis RD, Pereira EC, Pereira Maria IM, Soane AMNC, Silva JV. Significados, para os familiares, de conviver com um idoso com sequelas de acidente vascular cerebral (AVC). Interface (Botucatu, Online) [serial on the internet]. 2017 [cited $2017 \mathrm{Jul}$ 7];21(62):641-50. Available from: http:// www.scielo.br/pdf/icse/v21n62/1807-5762icse-1807-576220160206.pdf

15. Avelino ACA, Cunha ARR, Silva PMC, Azevedo EB, Silva JB, Ferreira FMO. 0 cuidado ao idoso portador de transtorno mental sob a ótica da família. Referência [serial on the internet]. 2013 [cited 2017 Jul 7];(9):75-83. Available from: $\quad$ http://www.scielo.mec.pt/pdf/ref/vserIIIn9/ serIIIn9a08.pdf

16. Medrano M, Rosario RL, Payano AN, Capellán NR. Burden, anxiety and depression in caregivers of Alzheimer patients in the Dominican Republic. Dement Neuropsychol [serial on the internet]. 2014 [cited 2017 Jul 7];8(4):84-8. Available from: $\quad$ http://www.scielo.br/pdf/dn/v8n4/1980-5764dn-08-04-00384.pdf

17. Vagetti GC, Oliveira V, Silva MP, Pacífico AB, Costa TRA, Campos W. Association of body mass index with the functional fitness of elderly women attending a physical activity program. Rev Bras Geriatr Gerontol [serial on the internet]. 2017 [cited 2017 Aug 4];20(2):214-24. Available from: http://www.scielo.br/pdf/rbgg/v20n2/1809-9823rbgg-20-02-00214.pdf

18. Valer DB, Aires M, Fengler FL, Paskulin LMG. Adaptation and validation of the Caregiver Burden Inventory for use with caregivers of elderly individuals. Rev Latinoam Enferm [serial on the internet]. 2015 [cited 2017 Aug 1];23(1):1308. Available from: http://www.scielo.br/pdf/rlae/ v23n1/0104-1169-rlae-23-01-00130.pdf

19. Santos FPA, Nery AA, Matumoto S. A produção do cuidado a usuários com hipertensão arterial e as tecnologias em saúde. Rev Esc Enferm USP [serial on the internet]. 2013 [cited 2017 Aug 1];47(1):107-14. Available from: http:// www.scielo.br/pdf/reeusp/v47n1/a14v47n1.pdf
20. Costa PCD, Francischetti GAPR, Pellegrino TV. Expectativa de enfermeiros brasileiros acerca do acolhimento realizado na atenção primária em saúde. Rev Salud Pública [serial on the internet]. 2016 [cited 2017 Aug 1];18(5):74655. Available from: https://revistas.unal.edu.co/index.php/ revsaludpublica/article/view/45304/62553

21. Maria INCB, Maria José MRL, Ylana CPL. A visita domiciliar como ferramenta de cuidado da fisioterapia na Estratégia Saúde da Família. Sanare (Sobral, Online) [serial on the internet]. 2015 [cited 2017 Aug 6];14(1)76-80. Available from: https://sanare.emnuvens.com.br/sanare/ article/view/612/329

22. Loureiro LSN, Fernandes MGM, Nóbrega MML, Rodrigues RAP. Sobrecarga em cuidadores familiares de idosos: associação com características do idoso e demanda de cuidado. Rev Bras Enferm [serial on the internet]. 2014 [cited 2017 Aug 3];67(2):227-32. Available from: http:// www.scielo.br/pdf/reben/v67n2/0034-7167reben-67-02-0227.pdf

23. Prato SCF, Andrade SM, Cabrera MAS, Dip RM, Santos HG, Dellaroza MSG, et al. Frequência e fatores associados a quedas em adultos com 55 anos e mais. Rev Saúde Pública [serial on the internet]. 2017 [cited 2017 Aug 4];51:37. Available from: http://www.scielo.br/pdf/rsp/v51/pt_00348910-rsp-S1518-87872017051005409.pdf

24. Gavasso WC, Beltrame V. Functional capacity and reported morbidities: a comparative analysis in the elderly. Rev Bras Geriatr Gerontol [serial on the internet]. 2017 [cited 2017 Aug 4];20(3):398-408. Available from: http:// www.scielo.br/pdf/rbgg/v20n3/1809-9823rbgg-20-03-00398.pdf
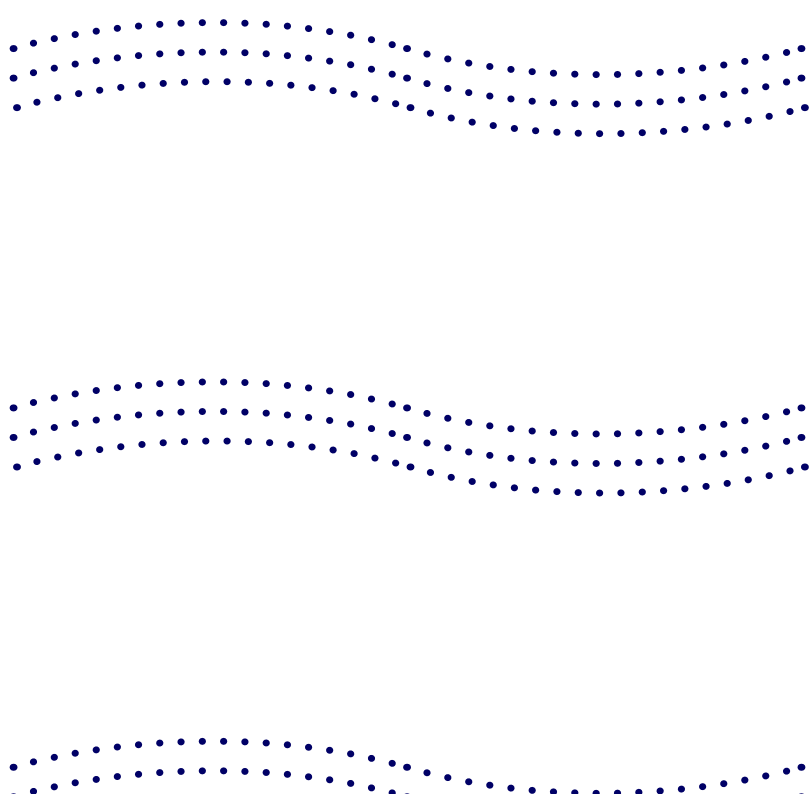
$\ldots \ldots \ldots \ldots \ldots \ldots \ldots \ldots \ldots \ldots \ldots \ldots \ldots \ldots \ldots \ldots$ $\ldots \ldots \ldots \ldots \ldots \ldots \ldots$

SANARE, Sobral - v.17, n.01,p.125-131, Jan./Jun. - 2018 - 131 